\title{
Synthesized Ceramide Induces Growth of Dermal Papilla Cells with Potential Contribution to Hair Growth
}

\author{
Jee Hye Oh, Kwan Ho Jeong, Jung Eun Kim, Hoon Kang \\ Department of Dermatology, St. Paul's Hospital, College of Medicine, The Catholic University of Korea, Seoul, Korea
}

Background: The ceramide is known to play an important role in the formation of intracellular lipids, and play a crucial role as a barrier for skin and hair cuticle. Recent study has revealed that ceramide has potential effect on hair growth in a mouse model. However, the role of ceramide in human dermal papilla cells (hDPCs) known to play an important role in hair growth is not well understood yet. Objective: The goal of this study was to investigate the effect of synthetic ceramides (oleyl and stearyl ceramides) on hair growth using hDPCs. Methods: hDPCs were treated with synthesized ceramides. hDPCs viability was evaluated by MTT assay. The expression of hair growth related factors were investigated by western blot, real-time polymerase chain reaction and growth factor array. The expression of $\beta$-catenin was confirmed by immunofluorescence. Results: Treatment with ceramides increased the expression of proteins affecting cell proliferation such as $\mathrm{BCl}-2, \mathrm{BAX}$, phosphorylated-ERK and Cyclin D1. Also, ceramides treatment were increased the expression of several growth factors, including epidermal growth factor family, and promote the expression of Wnt/ $\beta$-catenin and BMP2/4 signaling. Conclusion: Our data suggest that synthetic ceramides stimulates hair growth by induction proliferation of hDPCs via modulation of $\mathrm{Wnt} / \beta$ -catenin and BMP2/4 signaling. (Ann Dermatol 31(2) $164 \sim$

Received July 30, 2018, Revised October 16, 2018, Accepted for publication October 23, 2018

Corresponding author: Hoon Kang, Department of Dermatology, St. Paul's Hospital, College of Medicine, The Catholic University of Korea, 180 Wangsan-ro, Dongdaemun-gu, Seoul 02259, Korea. Tel: 82-2-958-2143, Fax: 82-2-969-8999, E-mail: johnkang@catholic.ac.kr ORCID: https://orcid.org/0000-0002-9822-4760

This is an Open Access article distributed under the terms of the Creative Commons Attribution Non-Commercial License (http://creativecommons. org/licenses/by-nc/4.0) which permits unrestricted non-commercial use, distribution, and reproduction in any medium, provided the original work is properly cited.

Copyright (c) The Korean Dermatological Association and The Korean Society for Investigative Dermatology
174, 2019)

-Keywords-

Ceramide, Dermal papilla cell, Hair, Wnt signaling pathway

\section{INTRODUCTION}

Hair aging is a complicated process involving various factors. The study of hair aging is divided into two main streams ${ }^{1}$. One is an intrinsic biological process at cellular level that is directly linked to hair growth. For hair growth and cycling, interaction between human dermal papilla cells (hDPCs) and mesenchymal stem cells in hair follicles and various growth factors are essential ${ }^{2}$. The other one is an extrinsic process that happens outside the scalp ${ }^{1}$. Extrinsic process is highly related to the cuticle layer. The cuticle exists at the outermost part of the hair. It protects the hair shaft from external mechanical stress. The cuticle layer is damaged by external stress such as ultraviolet rays, smoking, and malnutrition. It is known that when the cuticle layer is damaged, aging process of the hair can be initiated easily ${ }^{3}$.

In terms of structure of hair cuticle which protects hair from extrinsic stimuli, ceramide is the most important intracellular lipid that constitutes the lipid layer of hair cuticle along with fatty acid and cholesterol ${ }^{4}$. Ceramide is a component of intercellular lipids that are present in hair cuticles to protect and strengthen internal components of hair. Moreover, ceramide is a crucial second signal molecule that regulates various signaling pathways, including cell cycle, cellular senescence, and apoptosis ${ }^{5}$.

Previous studies have shown that ceramide has an antagonistic effects for cell growth and cell survival ${ }^{6}$. However, recently it has been reported that ceramide can affect hair 
loss prevention and hair growth enhancement ${ }^{7}$. In particular, ceramide synthase 4 (CerS4) plays an important role in the maintenance of epidermal stem cell homeostasis and regulation of the hair cycle by regulating Wnt signal and BMP signal ${ }^{8}$. Although ceramide can affect Wnt/BMP balance in $v_{i v o}{ }^{8}$, the effect of ceramide on growth pathway such as Wnt/BMP balance in vitro has not been confirmed.

Therefore, the objective of this study was to investigate the effect of ceramide on hDPCs at cellular level. To investigate the effect of existing natural $\mathrm{C} 8$-ceramide ( $\mathrm{N}$-octanoyl sphingosine) and ceramide-like synthetic compound on hair growth, we evaluated the hair growth pathway including Wnt/ $\beta$-catenin and BMP signaling changes after stimulation with ceramide. Three types of ceramide were used in this experiment: C8-ceramide and two newly synthetic ceramides (oleyl and stearyl ceramide). These newly synthesized ceramides are different from C8-ceramide in that they have higher solubility through double bonds and carbon chain length.

A
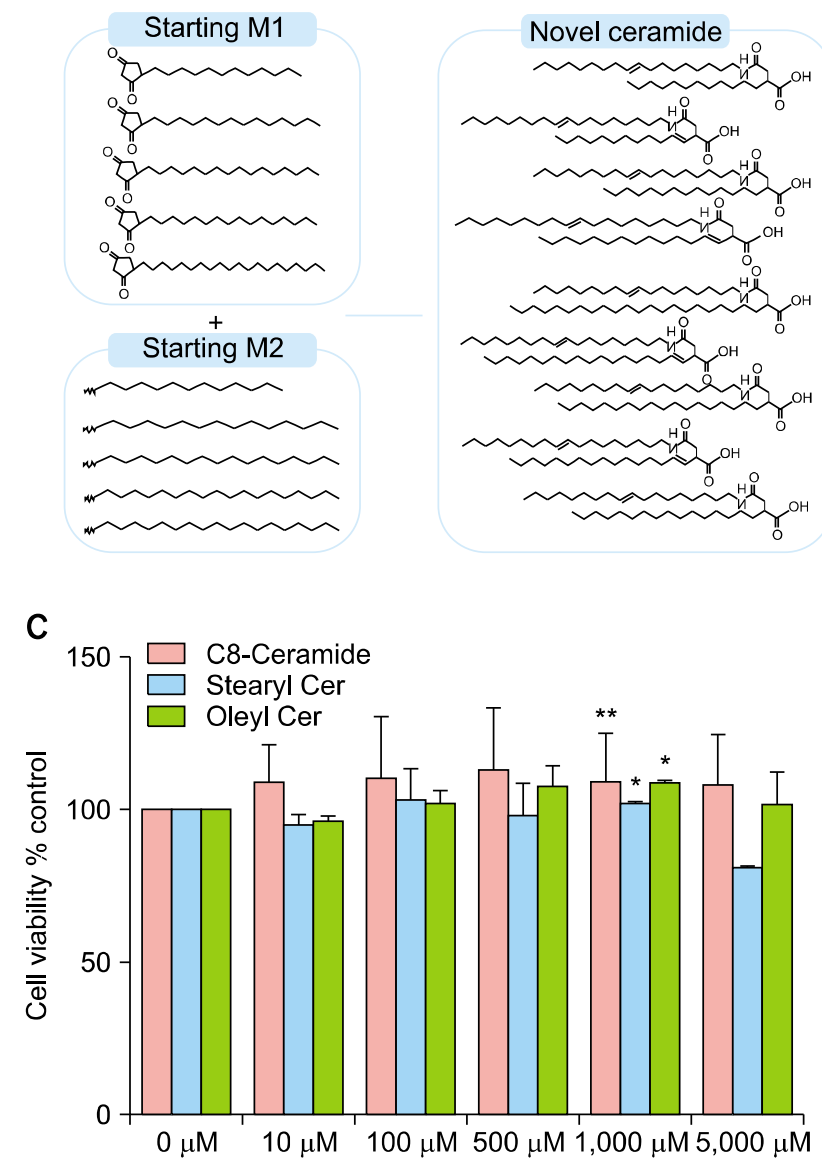

\section{MATERIALS AND METHODS}

\section{Ceramide material preparation}

C8-ceramide was purchased from Cayman Chemical (Ann Arbor, MI, USA). Oleyl ceramide was synthesized by reacting one equivalent of 2-dodecenyl succinic anhydride and oleyl amine in methylene chloride at room temperature (RT) for 30 minutes. The reaction mixture was then extracted with ethanol and water and recrystallized to a pale yellow paste, with a purity of $95 \%$ and a yield of $98 \%$. Structure of the compound was confirmed by ${ }^{1} \mathrm{H}-\mathrm{NMR}$ and LC-MSMS spectrometer. ${ }^{1} \mathrm{H}-\mathrm{NMR}$ spectra were recorded on a ZEOL $400 \mathrm{MHz}$ FT-NMR spectrometer in $\mathrm{CDCl}$. LC-MSMS spectra were recorded on a Shiseido HTS HPLC system and an AB SCIEX API 3200 triple quadruple mass spectrometer. LC-MSMS separations module with electrospray ionization was carried out in positive ion mode. Stearyl ceramide was synthesized in the same manner using stearyl amine (Fig. 1A). All ceramides were dissolved in ethanol to obtain concentration of $1 \mathrm{mM}$ and store at $-20^{\circ} \mathrm{C}$.

B

Oleyl ceramide

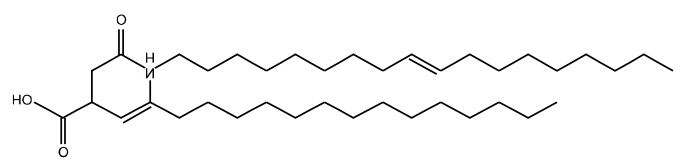

Stearyl ceramide

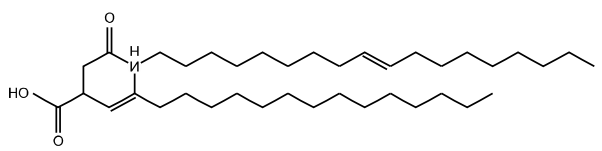

Fig. 1. Effects of ceramides on viability of human dermal papilla cells (hDPCs). (A) Scheme of ceramide's synthesis process. (B) Chemical structures of synthetic ceramides (oleyl and stearyl ceramide). (C) The effect of ceramides on hDPCs viability was determined by MTT assay. hDPCs were cultured for 24 hours. After starvation, these cultured hDPCs were treated with three kinds of ceramides for 72 hours. Stearyl Cer: stearyl ceramide, Oleyl Cer: oleyl ceramide. ${ }^{*} p<0.05$ compared with C8-ceramide treatment group, ${ }^{* *} p<0.01$ compared with group treated with C8-ceramide (positive control). 


\section{Cell culture}

hDPCs were purchased and cultured in Follicle Dermal papilla cell growth medium (PromoCell GmbH, Heidelberg, Germany) supplemented with $10 \%$ fetal bovine serum and $1 \%$ penicillin and placed in an incubator at $37^{\circ} \mathrm{C}$ with $5 \% \mathrm{CO}_{2}$.

\section{Cell viability assay (MTT assay)}

The viability of hDPCs was evaluated by 3-[4,5-dimethylthiazol-2-yl]-2,5-diphenyltetrazolium bromide (MTT) assay. Briefly, hDPCs were seeded into 24-well plates at density of $1.0 \times 10^{4}$ cell per well. After $48 \mathrm{~h}$ of incubation for hDPCs to reach confluency, three kinds of ceramides (C8-ceramide, oleyl, and stearyl ceramides) at various concentrations of $10 \mu \mathrm{M}, 100 \mu \mathrm{M}, 500 \mu \mathrm{M}, 1,000 \mu \mathrm{M}$ and 5,000 $\mu \mathrm{M}$ were used for treatment for 24 hours, 48 hours, and 72 hours under $1 \%$ penicillin conditions. After incubation, $100 \mu \mathrm{l}$ of MTT solution was added to each well and the hDPCs were then incubated at $37^{\circ} \mathrm{C}$ for 4 hours. After the medium was aspirated, $400 \mu$ I DMSO was added to each well. The absorbance of the plate was measured at wavelength of $540 \mathrm{~nm}$ using an ELISA plate reader.

\section{Real time polymerase chain reaction}

RNA was isolated from cultured hDPCs using TRIZOL reagent (Invitrogen, Carlsbad, CA, USA) and cDNA synthesis was performed using a cDNA synthesis kit (Takara, Shiga, Japan) according to the manufacturer's instructions. The cDNA was used for real-time polymerase chain reaction (PCR) which was carried out with SYBR Green (BioRad, Hercules, CA, USA). Sequences of primers used in real-time PCR are listed in Table 1.

\section{Western blot analysis}

The protocol for western blot analysis was described in a previous report ${ }^{9}$. Briefly, protein lysates from hDPCs were prepared using radio immunoprecipitation assay cell lysis buffer containing protease inhibitor cocktail. The cell lysate was collected by scraping after removed cell debris from lysed cells. Protein concentration was determined using a Bradford assay. Proteins were separated by $10 \%$ SDS-polyacrylamide gel electrophoresis and blotted onto polyvinylidene fluoride membrane. The membrane was blocked with 5\% BSA/TBST reagent for 1 hour at RT. It was then incubated with primary antibodies against total ERK, total Akt, phosphorylated-ERK, Akt, and GSK3 $\beta$, Cyclin-D1, $\beta$-catenin (Cell Signaling Technology, Beverly, MA, USA), Bcl-2, BAX, and $\beta$-actin (Santa Cruz Biotechnology Inc., Dallas, TX, USA) overnight at $4^{\circ} \mathrm{C}$ on a rotary shaker. The blotted membrane was then visualized with an AI600 Bio-Image analysis system (GE Healthcare Life Science, Chicago, IL, USA).

\section{Growth factor array}

Synthetic ceramides were screened for their effects on the expression of growth factors by using Human Growth Factor Antibody Array G1 (RayBiotech Inc., Norcross, GA, USA) in accordance with the manufacturer's protocol.

\section{Immunofluorescence staining}

Cultured hDPCs were seeded on 4-well chamber slides at density of $5 \times 10^{3}$ cells per well (SPL Life Science, Pocheon, Korea). After starvation, hDPCs were treated with C8-ceramide, oleyl, and stearyl ceramides at concentration of $1,000 \mu \mathrm{M}$ for 24 hours. After aspirating the medium, cells were rinsed with $1 \mathrm{X}$ PBS three times for $5 \mathrm{mi}$ -

Table 1. List of primers and real-time polymerase chain reaction conditions

\begin{tabular}{|c|c|c|c|}
\hline Species & Primer name & Forward & Reverse \\
\hline \multirow[t]{10}{*}{ Human } & $\beta$-catenin & TTTAAGCCTCTCGGTCTGTG & CAAATACCCTCAGGGGAACA \\
\hline & Wnt3 & CCTCAAGGACAAGTACGACA & GGCACCTTGAAGTAGGTGTA \\
\hline & Wnt5a & AATTCTGGCTCСАСTTGTTG & CAATTACAACCTGGGCGAAG \\
\hline & Wnt7a & TCTGTAACAAGATCCCAGGC & CATTGCGGAACTGAAACTGA \\
\hline & AP1 & TGTCCGAGAACTAAAGCCAA & AAAAGTCCAACGTTCCGTTC \\
\hline & Lef1 & CAGTGACGAGCACTTTTCTC & CGTGATGGGATATACAGGCT \\
\hline & Axin2 & CAGTGGATGCTGGAGAGTGA & TGCCAGTTTCTTTGGCTCTT \\
\hline & BMP2 & CAGGTCCTTTGACCAGAGTT & CCATGGTCGACCTTTAGGAG \\
\hline & BMP4 & ATTCCCGTCCAAGCTATCTC & CTACGGAATGGCTCCATAGG \\
\hline & GAPDH & GAAGGTGAAGGTCGGAGTCAA & GСTCCTGGAAGATGGTGATG \\
\hline
\end{tabular}

$\beta$-catenin: beta-catenin, Wnt3: wingless type MMTV integration site family, member 3, Wnt5a: wingless type MMTV integration site family, member 5A, Wnt7a: wingless type MMTV integration site family, member 7a, AP1: activator protein 1, Lef1: lymphoid enhancer-binding factor-1, Axin2: axin-related protein 2, BMP2: bone morphogenetic protein 2, BMP4: bone morphogenetic protein 4, GAPDH: glyceraldehydes 3-phosphate dehydrogenase. 
nutes each time. Then hDPCs were fixed with $4 \%$ paraformaldehyde for 15 minutes and permeabilized with $0.1 \%$ Triton X-100 for 10 minutes. After washing with 1X PBS, cells were blocked with $1 \%$ BSA in $1 \mathrm{X}$ PBS for 1 hour at RT on a shaker. They were incubated with anti-rabbit $\beta$ catenin (Cell Signaling Technology) primary antibody diluted at 1:100 in blocking buffer and incubated at $4^{\circ} \mathrm{C}$ overnight. On the next day, they were incubated with Alexa Fluor 488 labeled goat anti-rabbit secondary antibody (Invitrogen) diluted at 1:200 in PBST at RT for 1 hour in the dark. These stained cells were mounted with mounting medium containing DAPI to counterstain nuclei. Cells were then observed with a fluorescence microscope (Axiovert 200; Zeiss, Oberkochen, Germany).

\section{Statistical analysis}

All statistical data analyses were carried out using Graphpad Prism 5 Software. One-way analysis of variance (ANOVA) was used to evaluate significant difference at $p<0.05$.

\section{RESULTS}

\section{Viability of hDPCs after treatment with ceramides}

To investigate the effect of synthetic ceramides (Fig. 1B) on viability of hDPCs, we performed MTT assay. Firstly, optical concentrations of oleyl and stearyl ceramides and C8-ceramide as a positive control were determined. hDPCs were treated with various concentrations $(0 \mu \mathrm{M}, 10 \mu \mathrm{M}$, $100 \mu \mathrm{M}, 500 \mu \mathrm{M}, 1,000 \mu \mathrm{M}$, and 5,000 $\mu \mathrm{M})$ of oleyl and stearyl ceramides, and C8-ceramide for 72 hours. Results showed that oleyl and stearyl ceramides inhibited cell viability at 5,000 $\mu \mathrm{M}$ (Fig. 1C). However, viability of hDPCs was increased after treatment with C8-ceramide $(110 \%)$, oleyl ceramide $(109 \%)$, and stearyl ceramide (102\%) at 1,000 $\mu \mathrm{M}$. Based on these results, 1,000 $\mu \mathrm{M}$ was chosen as the proper concentration of ceramides for further experiments.

\section{Ceramides regulates $\mathrm{Bcl}-2 / \mathrm{BAX}$ and ERK/Cyclin-D1 in hDPCs}

We examined expression levels of Bcl-2 and BAX known as apoptosis regulator genes by western blot analysis. Results showed that $\mathrm{Bcl}-2$ expression was significantly enhanced by treatment with ceramides compare to that in the control group. However, treatment with ceramides showed no significant effect on BAX expression (Fig. 2A). When the ratio of $\mathrm{BCl}-2$ to $\mathrm{BAX}$ was compared, there was no significant difference between C8-ceramide treatment group and the control group. However, in groups treated with the two newly synthesized ceramides, the ratio of $\mathrm{BCl}-2$ to BAX was increased 1.22-fold in the group treated with oleyl ceramide and 1.53-fold in the group treated with stearyl ceramide. Such increases were statistically significant.

ERK is also an important factor involved in cell survival and proliferation ${ }^{10,11}$. We examined the effect of ceramides on phosphorylated-ERK in order to determine if our synthetic ceramides could affect mitogen-activated protein kinase (MAPK) signaling involved in the growth of hDPCs. Protein expression level of phosphorylated-ERK was increased following treatment with ceramides (Fig. 2B). PD98059, an ERK inhibitor, is known to regulate the proliferation of hDPCs. hDPCs were pretreated with ERK inhibitor PD98059 (10 $\mu \mathrm{M})$ for 1 hour and then incubated with ceramides for 24 hours.

Treatment of PD98059 reduced the overall activation of ERK compared to the untreated group. In Fig. 2C, phosphorylated-ERK expression was abolished in the PD98059 treated group compared to the untreated group. Also, we tested whether the ceramides affected phosphorylation tendency to aggravate. As a results, in the ceramide-treated group, the expression of phosphorylated-ERK was suppressed compared with Fig. 2B, but the expression was increased as compared with the control group.

Our results revealed that the expression level of Cyclin-D1 was increased after treatment with C8-ceramide, oleyl, and stearyl ceramide (Fig. 2D). These results indicate that our synthetic ceramides could regulate proliferation of hDPCs by regulating Bcl-2/BAX ratio and ERK/Cyclin-D1.

\section{Ceramide treatment upregulates growth factors including epidermal growth factor family}

Growth factors were assessed using growth factor membrane microarray (RayBiotech Inc.) according to the manufacturer's instructions. As shown in Fig. 3A, treatment with oleyl and stearyl ceramides increased growth factors levels overall. Epidermal growth factor (EGF) family members such as amphiregulin (AR), EGF, EGF receptor (EGFR), and insulin-like growth factor 1 (IGF-1) are known to stimulate nuclear translocation of $\beta$-catenin.

We confirmed that the IGF-1, EGF and EGFR expressions were increased in response to ceramides-treatment (Fig. 3B). AR expression was decreased by C8-cer treatment, but oleyl and stearyl ceramide-treatment increased $A R$ expression.

\section{Ceramides activates $\beta$-catenin pathway in hDPCs}

We focused on the $\beta$-catenin pathway involved in hair growth. The level of phosphorylated-Akt was significantly increased by treatment with ceramides compared to that in the control group (Fig. 4A). Our results also showed that oleyl and stearyl ceramides had a reversal effect on 
A
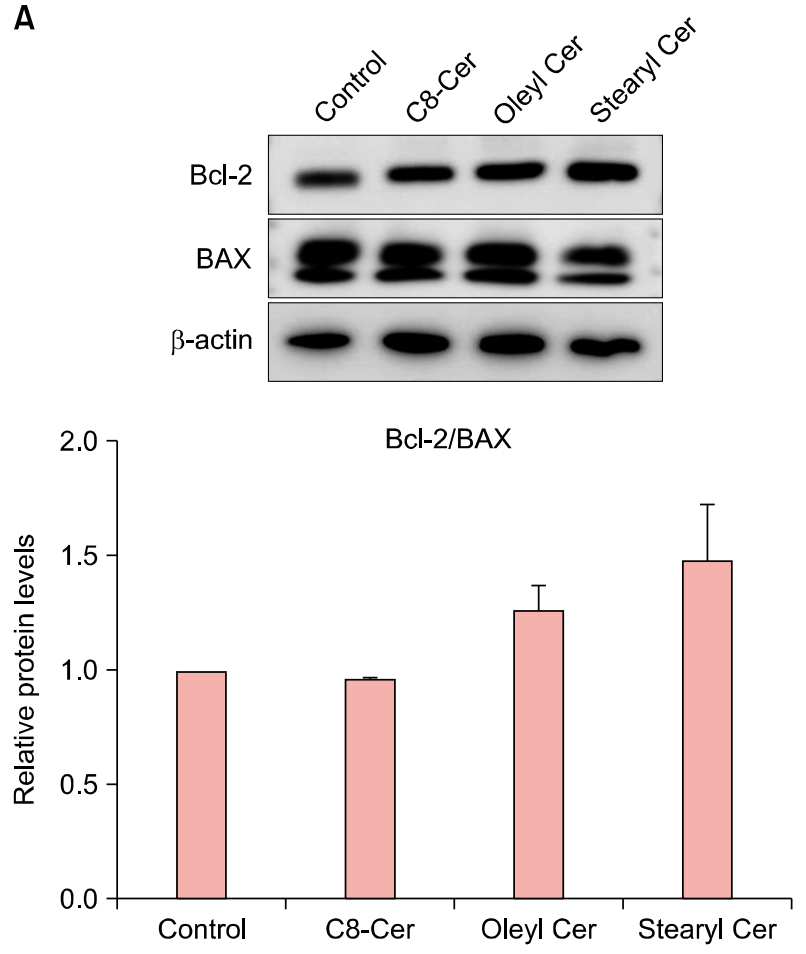

C
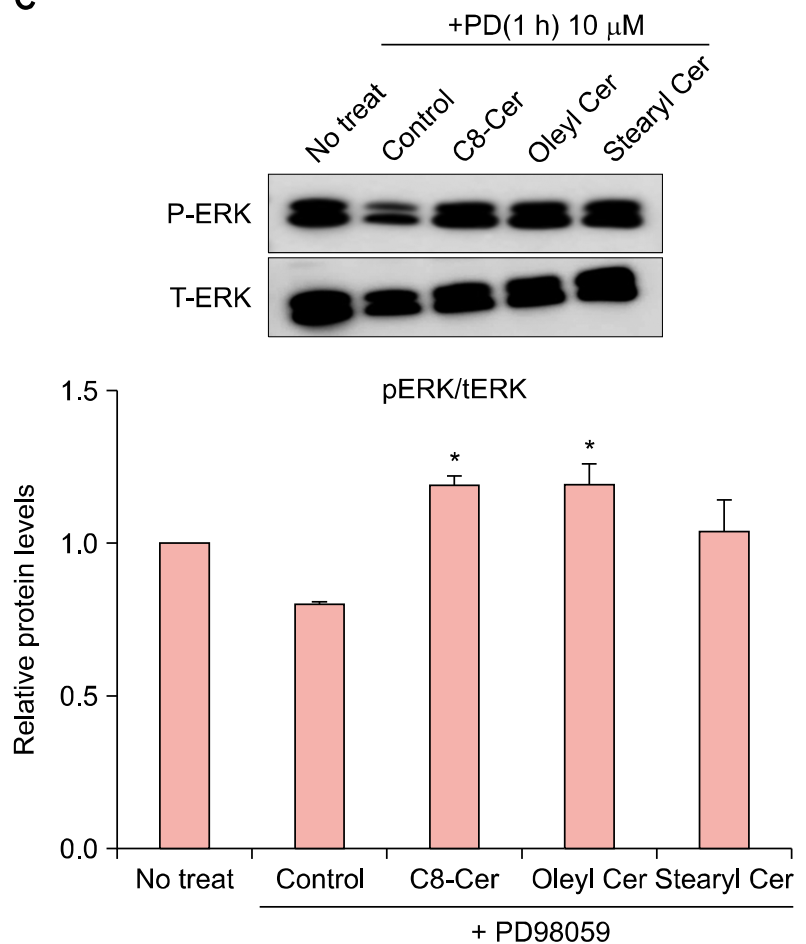

B
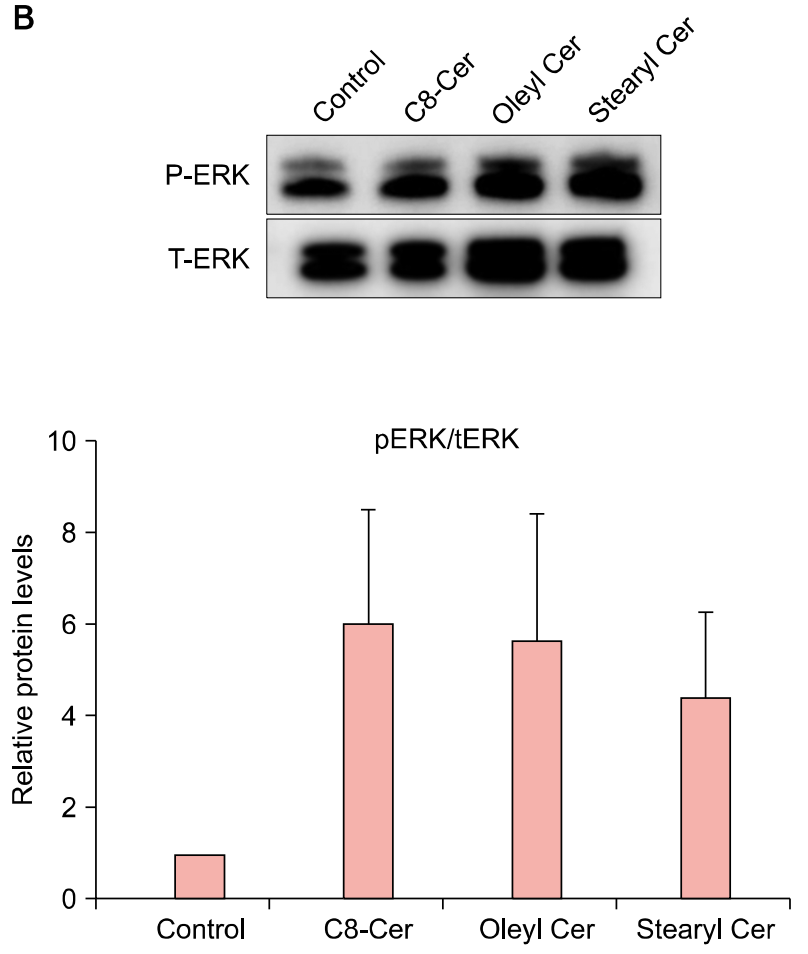

D
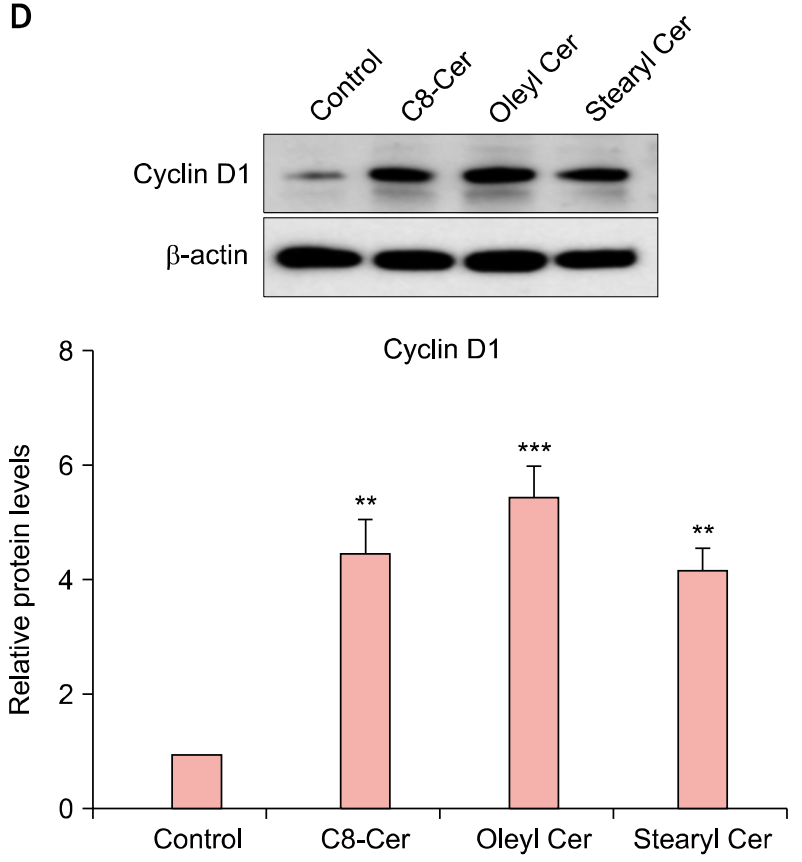

Fig. 2. Ceramide increases expression levels of Bcl-2/BAX and ERK/Cyclin-D1. (A) Expression levels of various factors known to affect proliferation were examined by western blot. Bcl-2 expression in groups treated with oleyl and stearyl ceramides was increased compare to those of the control group and C8-ceramide group. (B $\sim \mathrm{D})$ Phosphorylated-ERK was normalized relative to total ERK. Cyclin-D1 was normalized relative to $\beta$-actin. Western blot results showed upregulated levels of Cyclin-D1 and phosphorylated ERK after treatment with ceramides compare to the control group. Results are expressed as mean \pm standard deviation. C8-Cer: C8-ceramide, Stearyl Cer: stearyl ceramide, Oleyl Cer: oleyl ceramide. ${ }^{*} p<0.05$ compared with the control, ${ }^{* *} p<0.01$ compared with the control, ${ }^{* * *} p<0.001$ compared with the control. 
A

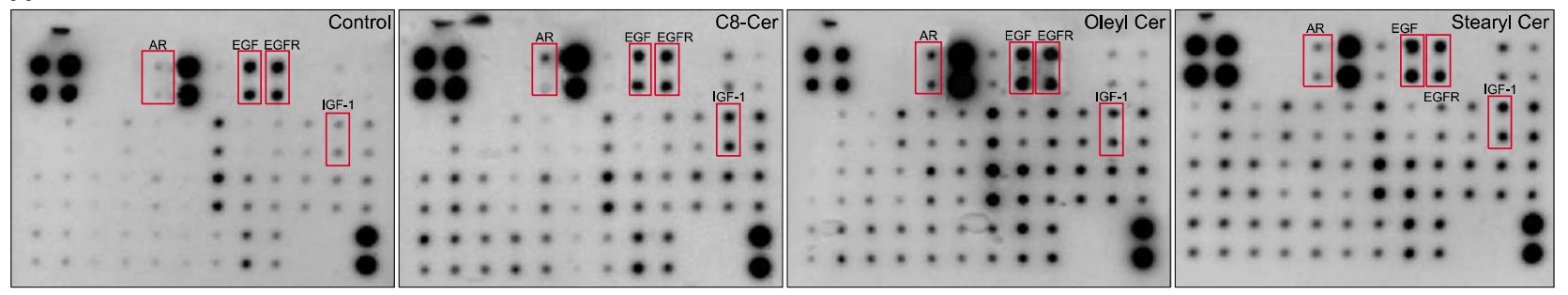

B
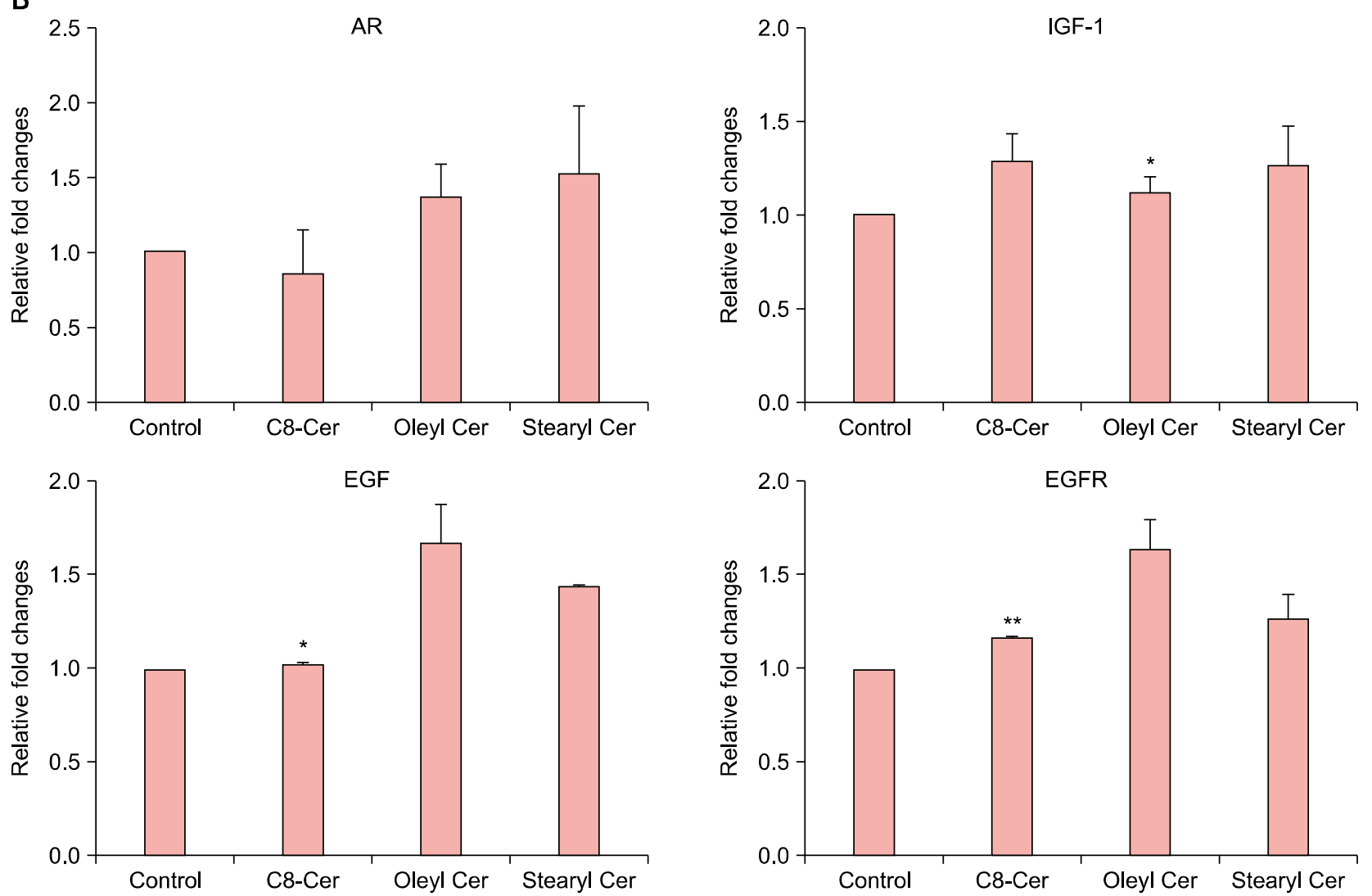

Fig. 3. (A) Ceramides upregulate growth factor protein levels. Human growth factor antibody array was used to detect growth factor expression in human dermal papilla cells (hDPCs). (B) Ceramide treatment significantly increased the expression of growth factors in hDPCs. Results are expressed as mean \pm standard deviation. C8-Cer: C8-ceramide, Stearyl Cer: stearyl ceramide, Oleyl Cer: oleyl ceramide, AR: amphiregulin, IGF-1: insulin-like growth factor 1, EGF: epidermal growth factor, EGFR: receptor.. ${ }^{*} p<0.05$ compared with the control, ${ }^{* *} p<0.01$ compared with the control.

LY294002, an Akt inhibitor. They induced a decrease in phosphorylation of Akt (Fig. 4A). Our results showed that protein expression level of phosphorylated-GSK3 $\beta$ was increased by treatment with ceramides including C8-ceramide, oleyl, and stearyl ceramides (Fig. 4B).

Western blot results showed that $\beta$-catenin expression was increased in groups treated with ceramides compared to that in the control group (Fig. 4B). Additionally, our immunofluorescent staining results confirmed the expression of $\beta$-catenin in the nucleus. Interestingly, the expression of $\beta$-catenin in the nucleus was very weak in the control group untreated with ceramides whereas the expression of $\beta$-catenin in the nucleus was significantly increased in groups treated with synthetic ceramides. In particular, its expression in the nuclei and cytoplasm in groups treated with synthetic ceramides (oleyl and stearyl ceramides) was significantly increased compared to that in the control group (Fig. 4C). These results suggest that our synthetic ceramides could induce $\beta$-catenin expression.

\section{Wnt $\beta$-catenin and BMP signaling in hDPCs}

Wnt signaling is known as the master regulator of hair 
A
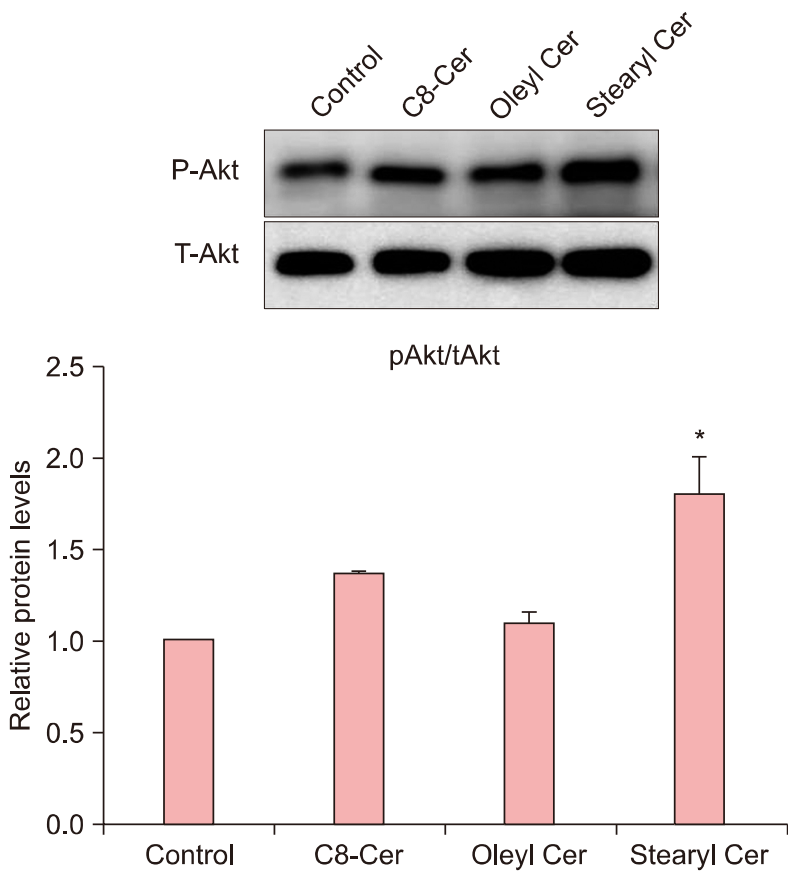

B
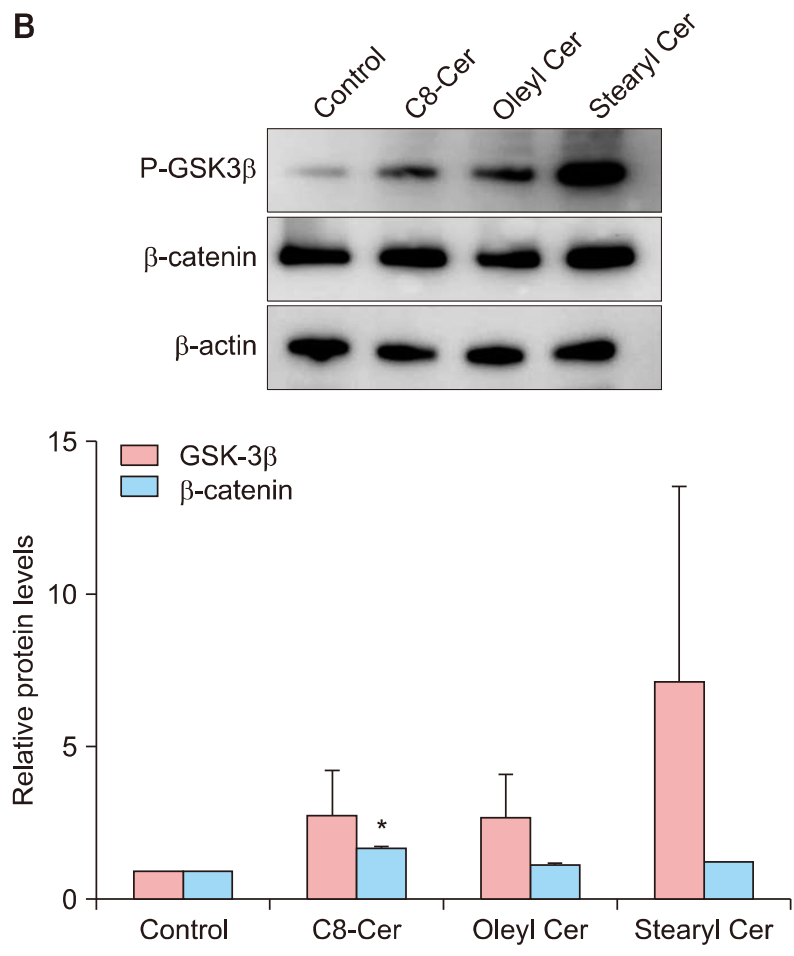

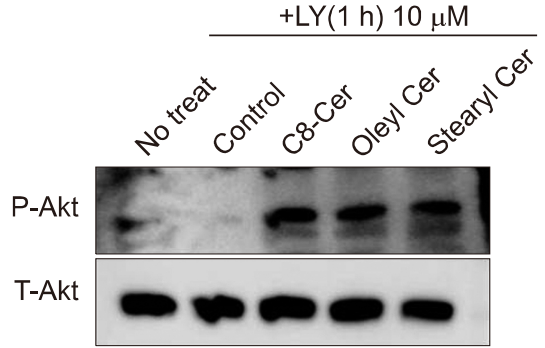

pAkt/tAkt

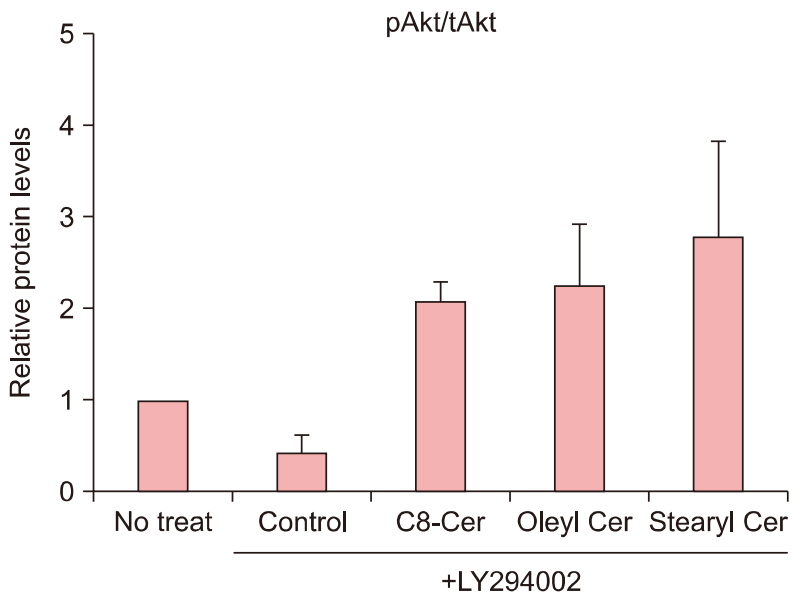

C

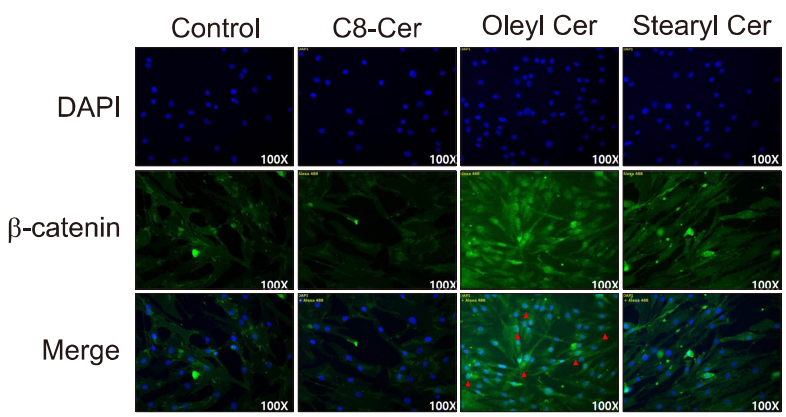

Fig. 4. Effects of ceramide treatment on B-catenin pathway. Phosphorylation of Akt/GSK3 $\beta$ and $\beta$-catenin expression was determined by western blot. Graph was schematized data of western blot band. (A, B) Phosphorylation of Akt/GSK3 $\beta$ and $\beta$-catenin expression was increased by ceramide treatment compare to those in the control. (C) $\beta$-catenin expression in the nucleus of human dermal papilla cells was determined by immunofluorescence staining. Oleyl and stearyl ceramides significantly increased $\beta$-catenin expression in nuclei compare to C8-ceramide treatment or control. DAPI (blue) was used to counterstain nuclei $(\times 100)$. Results are expressed as mean \pm standard deviation. C8-Cer: C8-ceramide, Stearyl Cer: stearyl ceramide, Oleyl Cer: oleyl ceramide. ${ }^{*} p<0.05$ compared with the control. 
growth and hair cycle while $\beta$-catenin is a key transcription factor activated by Wnt signaling ${ }^{12}$. BMP signaling is known to regulate stem cell activation during hair regeneration ${ }^{13}$. To determine whether our synthetic ceramides might affect $\mathrm{Wnt} / \beta$-catenin and BMP signaling, we conducted real-time PCR analysis. As shown in Fig. 5, treatment with stearyl ceramide increased Wnt signaling such as mRNA expression level of wnt3a (2.1-fold), wnt5a (1.8-fold), and wnt10b (6.1-fold) (Fig. 5A). Treatment with stearyl ceramide also significantly increased $\beta$-catenin signaling, including mRNA expression level of $\beta$-catenin (5.5-fold), AP-1 (3.2-fold), Lef-1 (3.8-fold), and Axin2 (2.2fold) compared to the control group (Fig. 5B). Moreover, treatment with oleyl ceramide increased mRNA expression level of wnt3a, wnt5a, and wnt7a (Fig. 5A), as well as $\beta$-catenin, AP-1, Lef-1, and Axin compared to the control group (Fig. 5B).

The mRNA expression of BMP2 and BMP4, which are known as BMP target gene, were downregulated by oleyl ceramide treatment. Stearyl ceramide treatment also induced a decrease in BMP4 expression (Fig. 5C). Our data support that our synthetic ceramides could regulate Wnt/ $\beta$-catenin pathway by inhibiting BMP target genes.

A

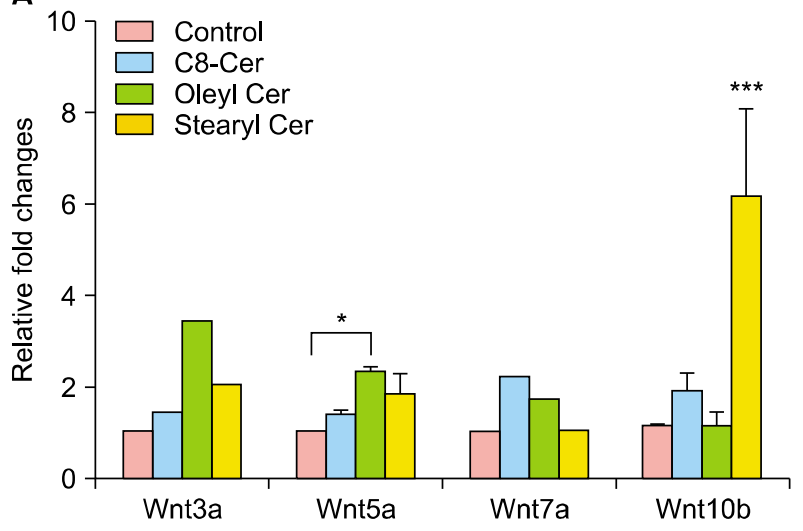

C

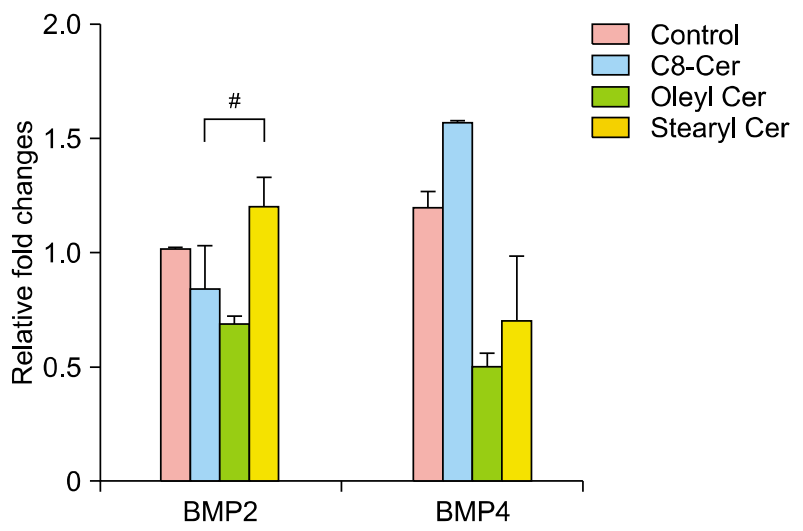

\section{DISCUSSION}

Ceramide is central component of cellular membrane. It is a key player in many signaling pathways involved in cell cycle arrest and differentiation ${ }^{14}$. Previous studies about effects of ceramide on hair have focused on developing cosmetics to protect hair shaft ${ }^{15}$. However, recent studies have suggested that ceramide promotes hair lengthening, leading to increased anagen follicles in mice and human organ culture system ${ }^{7}$. These results indicate that ceramide might not only play a role as hair shaft protector, but also act as hair growth stimulator. Consistent with previous studies, our results showed that the two newly synthesized ceramides could stimulate hair growth at cellular levels.

In the present study, we demonstrated that synthetic ceramides could increase proliferation of hDPCs accompanied by increased Bcl-2/BAX and phosphorylated-ERK/ Cyclin-D1 expression levels. We also observed that expression levels of $\beta$-catenin stimulated genes such as phosphorylated-Akt/GSK3 $\beta$ and AR/EGF/EGFR/IGF-1 were increased after treatment with ceramides. In addition, oleyl and stearyl ceramides could upregulate the expression of $\mathrm{Wnt} / \beta$-catenin signaling and downregulate BMP2 and

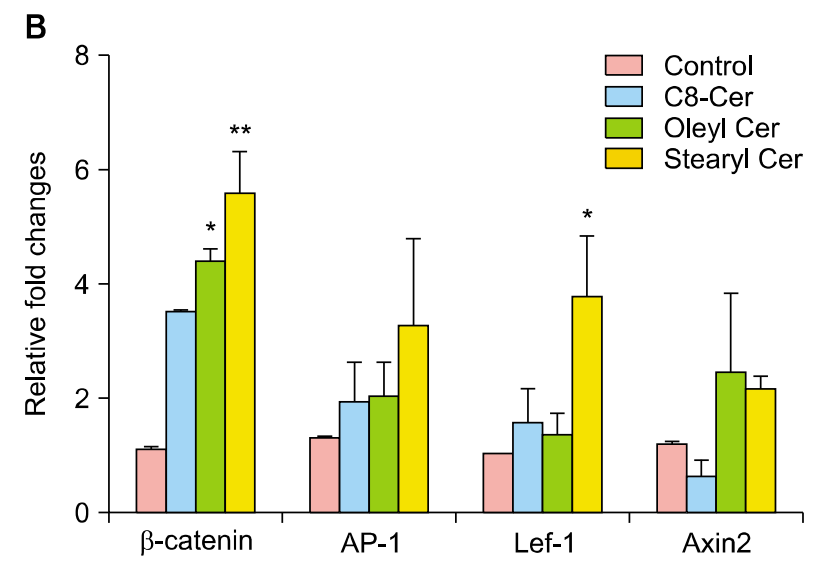

Fig. 5. Ceramides upregulate expression of $\mathrm{Wnt} / \beta$-catenin targets but downregulate expression of BMP target. Expression levels of target genes were determined using real-time polymerase chain reaction. (A) Wnt target genes: Wnt3, Wnt5a, Wnt7a, Wnt10b; (B) $\beta$-catenin target genes: $\beta$ catenin, AP-1, Lef-1, Axin2; and (C) BMP target genes: BMP2, BMP4. Results are expressed as mean \pm standard deviation. C8-Cer: C8-ceramide, Stearyl Cer: stearyl ceramide, Oleyl Cer: oleyl ceramide. ${ }^{*} p<0.05$ compared with the control, ${ }^{* *} p<0.01$, ${ }^{* * *} p<0.001$ compared with the control, ${ }^{*} p<0.05$ compared with the group treated with C8-ceramide. 
BMP4 (BMP2/4) expression. Taken together, our results suggest that synthetic ceramides have hair growth stimulating potentials.

Studies about hair growth have shown that the proliferation of hDPCs is accompanied by increased duration of anagen phase ${ }^{16}$. Hair follicle size is determined by the volume of hDPCs while hDPCs volume depends on the number of cells ${ }^{17}$. Hence, we evaluated the effect of synthetic ceramides on hDPCs proliferation. hDPCs were treated with various concentrations of ceramides. Our results confirmed that synthetic ceramides at 1,000 $\mu \mathrm{M}$ could increase viability of hDPCs. These results indicate that these synthetic ceramides might contribute to the proliferation of hDPCs.

Apoptosis is mainly processed through the intrinsic death pathway ${ }^{18}$. The most important regulators of apoptosis process are members of the $\mathrm{Bcl}-2$ gene family. $\mathrm{Bcl}-2$ protein is known to possess anti-apoptotic ability to obstruct a variety of apoptotic signals. BAX protein is another member of the $\mathrm{Bcl}-2$ family. It plays a pro-apoptotic role by stimulating apoptosis function, unlike $\mathrm{BCl}-2$ protein ${ }^{19}$. It has been reported that cellular $\mathrm{BCl}-2 / \mathrm{BAX}$ ratio represents regulation of cell survival ${ }^{20}$. Also, Bcl-2/BAX ratio was considered important in the hair cycle. It is known that the expression of $\mathrm{BCl}-2$ is dependent on the cycling of hair follicle, and apoptosis is the central program of the hair cycle ${ }^{21}$. Our results revealed that BAX expression did not change while Bcl-2 expression was significantly increased in groups treated with synthetic ceramides. Bcl-2/BAX ratio was increased in oleyl or stearyl ceramide treated group compared to that in the control group.

Ceramide activation may either activate or inhibit the MAPK pathway depending on the cell type ${ }^{22}$. MAPK signal is known to regulate various cellular responses such as cellular proliferation, cell differentiation, development, and apoptosis $^{23}$. ERK is known as one of MAPK signaling pathways involved in cell survival and growth ${ }^{10}$. Also, ERK pathway was known stimulate hDPCs proliferation ${ }^{24}$. CyclinD1 is also a key protein that promotes cell cycle (G1-S phase) progression. An increase in cyclin-D1 expression is required for cell proliferation to occur ${ }^{25}$.

In this study, we examined the effect of synthetic ceramides on phosphorylation of ERK and Cycin-D1 expression. Results showed that treatment with oleyl and stearyl ceramides increased phosphorylation of ERK and induced expression of Cyclin-D1 protein. Previous studies have shown that high concentration of ceramide in cells induces apoptosis ${ }^{6}$. However, our results showed Bcl-2/BAX ratio and ERK/Cyclin-D1 levels were increased by treatment with our synthetic ceramides. Although the underlying apoptotic effect of ceramide on hDPCs is not fully understood yet, our results show that synthetic ceramides may stimulate proliferation of hDPCs by regulating $\mathrm{Bcl}-2 /$ BAX ratio and ERK/Cyclin-D1 pathway.

Hair growth involves various transcription factors. EGF family members are known to stimulate nuclear translocation of $\beta$-catenin and upregulate the expression of Wnt10b and $\beta$-catenin levels. Especially, IGF-1 is known as an important effect on hair growth and extended the anagen phase generally ${ }^{26}$. The $\mathrm{Wnt} / \beta$-catenin signaling pathway is known as a master regulator for hair growth and hair follicle morphogenesis ${ }^{12}$. We investigated Akt/ $\beta$-catenin signaling pathway to elucidate the molecular mechanism involved in the effect of ceramides relate to $\beta$-catenin activation. To activate $\beta$-catenin translocation into nucleus, activation of Akt is needed. Akt is involved in signal transduction pathways such as inflammatory mediator, glycogen metabolism, and proliferation apoptosis ${ }^{27}$. Activation of Akt results in phosphorylation of several substrates such as GSK-3 $\beta$. GSK-3 $\beta$ is an active enzyme negatively regulated by Wnt pathway and Akt-induced phosphorylation. In the Wnt pathway, GSK-3 $\beta$ phosphorylation and inactivation will lead to the accumulation and nuclear translocation of $\beta$-catenin ${ }^{28}$. Activated $\beta$-catenin induces $W n$ signaling activation. Therefore, $\beta$-catenin is an essential molecule in Wnt signaling. As shown in Fig. 4 , ceramides-treatment increased phosphorylated GSK3 $\beta$ via activation of Akt. Finally, $\beta$-catenin expression in the nucleus was increased by ceramide-treatment. Our findings are consistent with results of Kwack et al. ${ }^{16}$ reporting that minoxidil can increase phosphorylation levels of Akt/ GSK3 $\beta$ and $\beta$-catenin expression.

The balance between $\mathrm{Wnt} \beta$-catenin pathway and BMP pathway is important for hair growth cycling ${ }^{29}$. Axin2 and Lef- 1 are directed target genes of the $\beta$-catenin pathway. Axin2 plays a role in regulating $\beta$-catenin level. Cytoplasmic protein is activated when Axin2 binds to $\beta$-catenin. Lef-1 is known as the binding site for $\beta$-catenin to accelerate directional differentiation of hair follicle stem cells ${ }^{30}$. Wnt3a and Wnt7a are inductive signals to maintain hDPCs in an anagen state ${ }^{31}$. Wnt5a is a key target gene of Shh in hair follicle morphogenesis ${ }^{32}$. Wnt10b, also known as hair follicle's growth stimulator, promotes hair follicle regrowth by upregulating $\mathrm{Wnt} / \beta$-catenin signaling pathway related factors including Axin2 and Lef- $1^{33}$. Previous studies have also reported that $\mathrm{Wnt} 10 \mathrm{~b} / \beta$-catenin signaling causes regeneration of hair follicles and proliferation of hair matrix cells $^{33,34}$. As shown in Fig. 5, treatment with synthetic ceramides upregulated expression of $\beta$-catenin target genes such as Lef-1, AP-1, and Axin2. Especially, Wnt10b and $\beta$ catenin expression levels were significantly increased by treatment with stearyl ceramide. These findings indicate 
that treatment with synthetic ceramides has potential to affect hair growth.

BMP signaling can promote telogen. It has been reported that inhibition of BMP signaling induces Wnt signaling and initiates hair growth early ${ }^{13}$. BMP2/4 activation can elaborately regulate the development of hair follicle bulge stem cell activation and growth during hair cycle ${ }^{35}$. As shown in Fig. 5, treatment with oleyl ceramide downregulated expression levels of BMP target genes (BMP2/4). These results suggest that downregulating BMP2/4 expression could help hair growth via early activation of Wnt signaling.

In conclusion, our study demonstrated that synthetic ceramides had positive effects on hair cell proliferation by regulating $\mathrm{Bcl}-2 / \mathrm{BAX}$ and ERK/Cyclin-D1 in hDPCs. Moreover, synthetic ceramides have potential to stimulate hair growth via Wnt/ $\beta$-catenin and BMP2/4 signaling in hDPCs. Our results provide novel information that synthetic ceramides such as oleyl and stearyl ceramides might have potential to help hair growth via cell proliferation by regulating gene expression in hDPCs. Although these findings are just laboratory results, our results explained the possibility of stimulating hair growth by treatment with ceramide. Furthermore, our synthetic ceramides might be used as new therapeutic agents to promote hair growth.

\section{ACKNOWLEDGMENT}

This research was supported by the Ministry of Trade, Industry \& Energy (MOTIE), Korea Institute for Advancement of Technology (KIAT) through the Encouragement Program for The Industries of Economic Cooperation Region (R0005754).

\section{CONFLICTS OF INTEREST}

The authors have nothing to disclose.

\section{ORCID}

Jee Hye Oh, https://orcid.org/0000-0002-7971-9936

Kwan Ho Jeong, https://orcid.org/0000-0002-3631-0866

Jung Eun Kim, https://orcid.org/0000-0003-1670-0995

Hoon Kang, https://orcid.org/0000-0002-9822-4760

\section{REFERENCES}

1. Trüeb RM. Oxidative stress in ageing of hair. Int J Trichology 2009;1:6-14.

2. Andl T, Reddy ST, Gaddapara T, Millar SE. WNT signals are required for the initiation of hair follicle development. Dev
Cell 2002;2:643-653.

3. Takahashi T, Mamada A, Breakspear S, Itou T, Tanji N. Age-dependent changes in damage processes of hair cuticle. J Cosmet Dermatol 2015;14:2-8.

4. Lee WS, Oh TH, Chun SH, Jeon SY, Lee EY, Lee $S$, et al. Integral lipid in human hair follicle. J Investig Dermatol Symp Proc 2005;10:234-237.

5. Sasaki T, Hazeki K, Hazeki O, Ui M, Katada T. Permissive effect of ceramide on growth factor-induced cell proliferation. Biochem J 1995;311:829-834.

6. Venable ME, Lee JY, Smyth MJ, Bielawska A, Obeid LM. Role of ceramide in cellular senescence. J Biol Chem 1995; 270:30701-30708.

7. Park BM, Bak SS, Shin KO, Kim M, Kim D, Jung SH, et al. Promotion of hair growth by newly synthesized ceramide mimetic compound. Biochem Biophys Res Commun 2017; 491:173-177.

8. Peters F, Vorhagen S, Brodesser S, Jakobshagen K, Brüning JC, Niessen CM, et al. Ceramide synthase 4 regulates stem cell homeostasis and hair follicle cycling. J Invest Dermatol 2015;135:1501-1509.

9. Jeong $\mathrm{KH}$, Joo HJ, Kim JE, Park YM, Kang H. Effect of mycophenolic acid on proliferation of dermal papilla cells and induction of anagen hair follicles. Clin Exp Dermatol 2015;40:894-902.

10. Li W, Man XY, Li CM, Chen JQ, Zhou J, Cai SQ, et al. VEGF induces proliferation of human hair follicle dermal papilla cells through VEGFR-2-mediated activation of ERK. Exp Cell Res 2012;318:1633-1640.

11. Han JH, Kwon OS, Chung JH, Cho KH, Eun HC, Kim KH. Effect of minoxidil on proliferation and apoptosis in dermal papilla cells of human hair follicle. J Dermatol Sci 2004;34: 91-98.

12. Rishikaysh P, Dev K, Diaz D, Qureshi WM, Filip S, Mokry J. Signaling involved in hair follicle morphogenesis and development. Int J Mol Sci 2014;15:1647-1670.

13. Zhang J, He XC, Tong WG, Johnson T, Wiedemann LM, Mishina $Y$, et al. Bone morphogenetic protein signaling inhibits hair follicle anagen induction by restricting epithelial stem/progenitor cell activation and expansion. Stem Cells 2006;24:2826-2839.

14. Uchida Y. Ceramide signaling in mammalian epidermis. Biochim Biophys Acta 2014;1841:453-462.

15. Méndez S, Manich AM, Martí M, Parra JL, Coderch L. Damaged hair retrieval with ceramide-rich liposomes. J Cosmet Sci 2011;62:565-577.

16. Kwack MH, Kang BM, Kim MK, Kim JC, Sung YK. Minoxidil activates $\beta$-catenin pathway in human dermal papilla cells: a possible explanation for its anagen prolongation effect. J Dermatol Sci 2011;62:154-159.

17. Elliott K, Stephenson TJ, Messenger AG. Differences in hair follicle dermal papilla volume are due to extracellular matrix volume and cell number: implications for the control of hair follicle size and androgen responses. J Invest Dermatol 1999;113:873-877.

18. Elmore S. Apoptosis: a review of programmed cell death. Toxicol Pathol 2007;35:495-516. 
19. Lutter M, Perkins GA, Wang X. The pro-apoptotic Bcl-2 family member $t B i d$ localizes to mitochondrial contact sites. BMC Cell Biol 2001;2:22.

20. Hanada M, Aimé-Sempé C, Sato T, Reed JC. Structurefunction analysis of $\mathrm{BCl}-2$ protein. Identification of conserved domains important for homodimerization with $\mathrm{BCl}-2$ and heterodimerization with Bax. J Biol Chem 1995;270: 11962-11969.

21. Stenn KS, Lawrence L, Veis D, Korsmeyer S, Seiberg M. Expression of the bcl-2 protooncogene in the cycling adult mouse hair follicle. J Invest Dermatol 1994;103:107-111.

22. Mathias S, Peña LA, Kolesnick RN. Signal transduction of stress via ceramide. Biochem J 1998;335:465-480.

23. Zhang W, Liu HT. MAPK signal pathways in the regulation of cell proliferation in mammalian cells. Cell Res 2002;12: 9-18.

24. Joo HJ, Jeong KH, Kim JE, Kang H. Various wavelengths of light-emitting diode light regulate the proliferation of human dermal papilla cells and hair follicles via Wnt/ $\beta$-catenin and the extracellular signal-regulated kinase pathways. Ann Dermatol 2017;29:747-754.

25. Yang $\mathrm{K}$, Hitomi $M$, Stacey DW. Variations in cyclin D1 levels through the cell cycle determine the proliferative fate of a cell. Cell Div 2006;1:32.

26. Ahn SY, Pi LQ, Hwang ST, Lee WS. Effect of IGF-I on hair growth is related to the anti-apoptotic effect of IGF-I and up-regulation of PDGF-A and PDGF-B. Ann Dermatol 2012; 24:26-31.

27. Liu $\mathrm{P}$, Cheng $\mathrm{H}$, Roberts TM, Zhao JJ. Targeting the phosphoinositide 3-kinase pathway in cancer. Nat Rev Drug Discov 2009;8:627-644.

28. Monick MM, Carter AB, Robeff PK, Flaherty DM, Peterson
MW, Hunninghake GW. Lipopolysaccharide activates Akt in human alveolar macrophages resulting in nuclear accumulation and transcriptional activity of beta-catenin. J Immunol 2001;166:4713-4720.

29. Kandyba E, Leung Y, Chen YB, Widelitz R, Chuong CM, Kobielak K. Competitive balance of intrabulge BMP/Wnt signaling reveals a robust gene network ruling stem cell homeostasis and cyclic activation. Proc Natl Acad Sci U S A 2013;110:1351-1356.

30. DasGupta R, Kaykas A, Moon RT, Perrimon N. Functional genomic analysis of the Wnt-wingless signaling pathway. Science 2005;308:826-833.

31. Kishimoto J, Burgeson RE, Morgan BA. Wnt signaling maintains the hair-inducing activity of the dermal papilla. Genes Dev 2000;14:1181-1185.

32. Reddy S, Andl T, Bagasra A, Lu MM, Epstein DJ, Morrisey $E E$, et al. Characterization of Wnt gene expression in developing and postnatal hair follicles and identification of Wnt5a as a target of Sonic hedgehog in hair follicle morphogenesis. Mech Dev 2001;107:69-82.

33. Ouji Y, Yoshikawa M, Shiroi A, Ishizaka S. Promotion of hair follicle development and trichogenesis by Wnt-10b in cultured embryonic skin and in reconstituted skin. Biochem Biophys Res Commun 2006;345:581-587.

34. Li YH, Zhang K, Ye JX, Lian XH, Yang T. Wnt10b promotes growth of hair follicles via a canonical Wnt signalling pathway. Clin Exp Dermatol 2011;36:534-540.

35. Plikus MV, Mayer JA, de la Cruz D, Baker RE, Maini PK, Maxson R, et al. Cyclic dermal BMP signalling regulates stem cell activation during hair regeneration. Nature 2008; 451:340-344. 